\title{
Reward reactivity and dark flow in slot-machine gambling: "Light" and "dark" routes to enjoyment
}

\author{
MIKE J. DIXON ${ }^{1,2}$, JEFFREY GUTIERREZ ${ }^{2}$, CHANEL J. LARCHE ${ }^{1,2}$, MADISON STANGE $^{1,2}$, \\ CANDICE GRAYDON ${ }^{1,2}$, TYLER B. KRUGER ${ }^{1,2}$ and STEPHEN D. SMITH ${ }^{3}$ \\ ${ }^{1}$ Department of Psychology, University of Waterloo, Waterloo, ON, Canada \\ ${ }^{2}$ Gambling Research Lab, University of Waterloo, Waterloo, ON, Canada \\ ${ }^{3}$ Department of Psychology, University of Winnipeg, Winnipeg, MB, Canada
}

(Received: March 25, 2019; revised manuscript received: July 2, 2019; accepted: July 2, 2019)

\begin{abstract}
Background and aims: Slot machines are a very popular form of gambling. In this study, we look at two different routes to enjoying slots play. One route involves the degree to which players react to rewards. The other route involves what we call dark flow - a pleasurable, but maladaptive state where players become completely engrossed in slots play, providing an escape from the depressing thoughts that characterize their everyday lives. Methods: One hundred and twenty-nine high-frequency slots players were tested on slot-machine simulators set up in the lobby of a casino. We measured reward reactivity using post-reinforcement pauses (PRPs) and the force with which players pressed the spin button following different slot-machine outcomes. For each player, we calculated the slopes of PRPs and force as a function of credit gains. We also assessed players' slots game enjoyment and their experience of dark flow, depression, and problem gambling. Results: Both the PRP and the force measures of reward reactivity were significantly correlated with players' enjoyment of the slots session, but neither measure was correlated with either problem gambling or depression. Ratings of dark flow were strongly correlated with slots enjoyment (which accounted for far more positive affect variance than the reward reactivity measures) and were correlated with both problem gambling scores and depression. Discussion and conclusions: Our results suggest that of these two routes to enjoying slot-machine play, the dark flow route is especially problematic. We contend that the dark flow state may be enjoyable because it provides escape from the negative thoughts linked to depression.
\end{abstract}

Keywords: mindfulness, depression, dark flow, mind-wandering, gambling

\section{SLOT-MACHINE GAMBLING: "LIGHT" AND "DARK" PATHWAYS TO ENJOYMENT}

For most gamblers, slot machines are a means of entertainment, and their appeal can be linked to a number of features. First, unlike many forms of gambling such as traditional lotteries, players do not have to wait long to find out whether they have won or lost - they spin and the machine's feedback is practically immediate. Second, when players spin and win, monetary gains are accompanied by high-fidelity attention-grabbing music and amusing animations (Griffiths \& Parke, 2005; Haas \& Edworthy, 1996). Third, players never know when wins will occur - wins and losses on slot machines are offered to the player using a variable-ratio reinforcement schedule that makes the timing of the wins unpredictable (Haw, 2008). Finally, win sizes can vary tremendously, ranging from values just above the spin wager to jackpots that can total many thousands of dollars.

Although a majority of gamblers are recreational players who engage in gambling as a form of harmless entertainment, a small but significant subset of gamblers can experience severe gambling-related problems (Blaszczynski,
Sharpe, Walker, Shannon, \& Coughlan, 2005). Problems range from mounting financial debt, issues with interpersonal relationships, professional difficulties, and sometimes even involvement in criminal activities to support their gambling behavior (Lahn, 2005). To mitigate such gambling harms, it is imperative to understand the allure of slot machines, and more importantly, what aspects of slots play may be particularly problematic.

A significant reinforcer of gambling behavior, especially during slot-machine play, is physiological arousal (Coventry \& Constable, 1999; Coventry \& Hudson, 2001; Dixon et al., 2011; Lole, Gonsalvez, Blaszczynski, \& Clarke, 2011; Sharpe, Tarrier, Schotte, \& Spence, 1995). Indeed Brown, underscored its significance by calling it “... a major, if not the major reinforcer of gambling behavior for regular gamblers" (Brown, 1986, p. 1001). During a slot-machine session, players' arousal levels have been shown to fluctuate according to the outcomes they

\footnotetext{
* Corresponding author: Mike J. Dixon; Department of Psychology, University of Waterloo, 200 University Avenue West, Waterloo, ON N2L 3G1, Canada; Phone: +1 5198884567 ext. 32877; Fax: +1 519 746 8631; E-mail: mjdixon@uwaterloo.ca
}

This is an open-access article distributed under the terms of the Creative Commons Attribution-NonCommercial 4.0 International License, which permits unrestricted use, distribution, and reproduction in any medium for non-commercial purposes, provided the original author and source are credited, a link to the CC License is provided, and changes - if any - are indicated. 
experience. Specifically, players typically demonstrate lower arousal levels after experiencing losses, and higher arousal levels following wins (Coventry \& Constable, 1999; Coventry \& Hudson, 2001; Dixon, Harrigan, Sandhu, Collins, \& Fugelsang, 2010). Importantly, there appears to be a systematic relation between win size and arousal level, with larger wins typically leading to more arousal than smaller wins (Dixon et al., 2011; Lole et al., 2011).

Although there is a general consensus that arousal is a consequence and reinforcer of gambling behavior (and indeed seems to be an intrinsic part of why players find it exciting and enjoyable), it is less clear how arousal relates to problem gambling. Anderson and Brown (1984) showed higher trait-levels of arousal among problem gamblers than non-problem gamblers in a casino. Other studies, however, fail to report such arousal differences (Coulombe, Ladouceur, Desharnais, \& Jobin, 1992; Coventry \& Constable, 1999; Coventry \& Norman, 1998; Sodano \& Wulfert, 2010). Still, research demonstrates that any relation between gambling status and arousal may depend on how arousal is measured. Diskin and Hodgins (2003) found no differences in heart rate or skin conductance between problem and non-problem gamblers but found that problem gamblers reported greater subjective arousal than nonproblem gamblers. Similarly, Meyer et al. (2004) compared problem and non-problem gamblers reactions to playing blackjack at a casino in which they compared gambling sessions with non-gambling baseline periods. They showed no interaction involving gambling status with heart rate (both groups showed an elevation during gambling), but showed an interaction with cortisol levels. Given that cortisol levels track arousal, the authors concluded that problem gamblers showed greater arousal during gambling than did non-problem gamblers.

Other research has shown that problem gamblers have dampened reactions to gambling scenarios. Paris, Franco, Sodano, Frye, and Wulfert (2010) found that problem gamblers had an attenuated cortisol release in response to viewing gambling scenes. Since non-problem gamblers showed the expected increases in cortisol release when viewing these same videos, these researchers concluded that, as in other forms of addiction with repeated exposure, problem gamblers develop a tolerance, leading to a hypo-arousal response to gambling scenarios. Thus, while it appears that arousal is one of the keys to enjoying slots, the literature is mixed when assessing whether gambling status is linked to hyper- or hypo-arousal responses during gambling.

Importantly, arousal may not be the only reason people enjoy playing slots. A significant percentage of players gamble as a means of coping with painful emotional experiences often attributable to depressive or anxious symptomatology (Abbot \& Volberg, 1996; Getty, Watson, $\&$ Frisch, 2000). It may be that the continuous nature of slotmachine play, and the attention-capturing, intermittent rewards, prevents players from thinking about the negative aspects of their lives. Thus, it is this relief from such negative thoughts that they find enjoyable. This intense absorption into the gambling activity and disassociation from the outside world has been coined the "slot-machine zone" by players (Schüll, 2012), dissociation (Diskin \&
Hodgins, 1999, 2001; Jacobs, 1988; Schüll, 2005, 2012; Walker, 1992), and absorption or immersion (Dixon, Graydon, et al., 2014). Despite the subtle differences between terminology, it seems clear that some gamblers experience a state of hyper-focused attention while playing these machines. In addition, this subset of players tends to experience more gambling problems than those who do not enter this state of immersion (Murch, Chu, \& Clark, 2017). A comprehensive overview of this highly immersive state and its relation to problem gambling can be found in the review by Schluter and Hodgins (2019).

Dixon, Stange, et al. (2017) have referred to this state as "dark" flow because entering this state can have profound negative consequences for the player, such as spending more time than planned playing slot machines, and facing mounting financial consequences. Despite its negative consequences, this state is very similar to flow states described in positive psychology: the state involves distortions in the passage of time, intense focus on a given task, and freedom from distractions (Csikszentmihalyi, 1992). People who experience this state find it immensely pleasurable. Slot machines, especially those that allow multiline play, appear to be especially good at inducing this dark flow state for some gamblers (Dixon, Graydon, et al., 2014; Murch et al., 2017). Dixon, Graydon, et al. (2014) found that those players at a higher risk of gambling problems [as measured by the Problem Gambling Severity Index (PGSI)] more strongly endorsed flow item questions from the Game Experiences Questionnaire (GEQ; IJsselsteijn, de Kort, Poels, Jurgelionis, \& Bellotti, 2007) than those players at lower risk of gambling problems. In this study, Dixon et al. measured (dark) flow using the two flow items from the brief version of the Game Experience Questionnaire: "I forgot everything around me" and "I felt completely absorbed." More recently, Dixon, Stange, et al. (2017) replicated the correlation between PGSI scores and the degree to which players endorsed flow items from the GEQ. In that study, they used all five flow items from the full version of the GEQ: "I was fully occupied with the game," "I forgot everything around me," "I lost track of time," "I was deeply concentrated in the game," and "I lost connection with the outside world."

Dixon et al. (2019) suggested that having mindfulness problems outside of the gambling context may be related to dark flow experiences within the gambling context. They suggest that players' mentations in everyday life are characterized by habitual bouts of mind-wandering, but during slots play their attention is reined in by the attention-capturing sights and sounds of the machine. Thus, it is this intermittent reinforcement in which there is an exogenous capturing of attention, which induces a state of dark flow. Accordingly, context is important in accounting for the propensity of problem players to more strongly endorse flow-like states during slots play. Their mind-wandering prevents them from ever experiencing flow in everyday life, but during slots play, the exogenous reining in of attention by the machine induces an usual state for them - what they call the slot-machine zone, and what Dixon and colleagues call dark flow. In support of their contentions, they showed that problems with mindfulness outside of the slots machine context predicted the degree to which players experienced dark flow during a slots session. 
Importantly, they also showed that dark flow ratings were positively correlated with how much they enjoyed play during the session - the greater the ratings of dark flow, the higher the ratings of positive affect during the session.

In the current experiment, we seek to demonstrate that the arousal associated with reward reactivity and dark flow differentially impacts the enjoyment experienced during slots play. In order to provide empirical evidence for these two routes to enjoyment of slots play, particular attention must be paid to the measures used. Although physiological measures such as skin conductance, heart rate, and cortisol measurements are suitable for measuring arousal, the accompanying electrode wires and swabs used to assess physiological changes likely interfere with entering the dark flow state. We propose two less intrusive in-game measures: post-reinforcement pauses (PRPs) and the force with which players press the spin button to initiate spins.

\section{Measuring reward reactivity through PRPs}

In slots play, a PRP is the temporal duration between when an outcome (win or loss) is revealed and when the next spin is initiated (Dixon, MacLaren, Jarick, Fugelsang, \& Harrigan, 2012). Multiple studies have shown that the duration of a PRP is significantly longer after a winning outcome compared to a losing outcome (Dixon et al., 2010, 2012; Dixon, Larche, et al., 2017; Dixon \& Schreiber, 2004). In addition, studies have found that the greater the payout, the longer the PRP (Delfabbro \& Winefield, 1999; Dixon et al., 2012; Dixon, Larche, et al., 2017). The rationale used to explain this occurrence is that the momentary enjoyment of the reward temporarily inhibits the need for further reward seeking; therefore, the larger the reward, the larger the enjoyment and the greater the tendency to inhibit the reward-seeking behavior (Dixon et al., 2012; Leslie, 1996).

\section{Measuring reward reactivity through force}

Another means of indexing the hedonic excitement associated with wins is to measure the force with which players initiate spins following various outcomes. Since wins elicit high levels of excitement-induced arousal, players will typically initiate the next spin with more force following a win than a loss. In two recent studies (Dixon, Larche, et al., 2017; Dixon, Stange, et al., 2017), we showed that the larger the win size, the greater the force applied to trigger the next spin. Such a force measure may be a particularly effective way of measuring excitement and arousal in slot-machine gambling. Unlike skin conductance or heart rate, it does not require electrodes and cumbersome wires. Additionally, by mounting a force transducer under the spin button, measurements of force can be gathered without distracting the player from game play and compromising measures of dark flow.

\section{Present experiment}

The present experiment will use a combination of in-game measures of reward reactivity (PRPs and force) and retrospective self-reports of enjoyment and dark flow to discriminate between two potential pathways to slots enjoyment. To capture reward reactivity, we measured PRPs as a function of win sizes during a slot-machine playing session for each player. We predicted that those players who find wins highly rewarding will pause longer prior to initiating the next spin. Since larger wins are more rewarding than smaller wins, we predict a positive slope of PRP length when plotted over win size, with the magnitude of this slope indicating the degree to which the player positively reacts to the reward. Similarly, those who find wins exciting and arousing should press the spin button harder following wins than following losses. Since larger wins are more exciting and arousing than smaller wins, we predict greater force as a function of win size, with slopes increasing for players who react more positively to rewards. In sum, if each of these measures captures excitement and arousal-based reward, then those who show steeper slopes should be players who retrospectively report enjoying the playing sessions more. Therefore, we hypothesize a positive correlation between positive affect during play and each of our in-game measures of reward reactivity. Importantly, if wins are equally rewarding to gamblers as a whole (both recreational and problem players), then there is no reason to expect that PRPs as a function of win size, or force as a function of win size, would predict either problem gambling symptoms or depressive symptomatology.

In contrast to this non-problematic means of enjoying slots, some players will enjoy playing slots not for the excitement of the wins, but rather for the relief found in the state of dark flow. We hypothesize that players who enter this state of dark flow will report positive affect during slots play, but for different reasons than the reward associated with wins. For these players, we predict a positive correlation between dark flow and positive affect. Commensurate with the problematic nature of gambling to escape, however, we predict dark flow ratings to correlate positively with problem gambling status and depression scores.

The reward reactivity (PRP and force as a function of win size) measures were extracted from the data of participants reported in Dixon et al. (2019), which also reported a correlation between dark flow and positive affect during slots play. In this study, we use the newly extracted measures (PRP and force measures) to show that reward reactivity will account for aspects of slots enjoyment that are different from the enjoyment attributable to dark flow.

\section{Participants}

A total of 129 (76 males and 53 females) participants were recruited from Casino Brantford, in Brantford, Ontario, Canada. Participants were prescreened during recruitment to ensure that they were all 19 years of age or older (the legal age to play a slot machine in Ontario), were not in treatment for problem gambling, and played slot machines at least once a week. The age of the sample ranged from 19 to 89 years and averaged to 60.1 years of age $(S D=14.05)$. In exchange for participating in the experiment, each participant received a $\$ 25.00$ Walmart gift card and any winnings gained at the end of the slot-machine session.

\section{Apparatus}

Slot-machine simulator. Participants played a 5-reel multiline slot-machine simulator modeled after a commercially 
available multiline slot-machine game. Participants bet 1 credit per line on each of the 20 lines for a total bet of 20 credits per spin. This simulator has been used in previous studies and encapsulates many of the features of a modern multiline slot machine (e.g., Dixon, Graydon, et al., 2014). For instance, losing outcomes were followed by a complete absence of feedback (no animations or sounds). Winning outcomes (where the slot machine paid more than the spin wager) were accompanied by auditory celebratory jingles, and, as with commercially available machines, the length of this auditory feedback was proportional to win size. Winning outcomes also showed animations, which highlighted the line (or lines) responsible for a win. For example, if a winning line involved three scimitars during the celebratory animation, the scimitars would begin to spin. These animations would continue until the player triggered the next spin. Players did not have to wait until the auditory feedback finished playing but could interrupt the celebratory feedback by pressing the spin button, which immediately initiated the next spin. The simulator also featured Losses Disguised as Wins (LDWs), where the total number of credits gained was less than the spin wager. The playing session consisted of 301 spins, containing 202 losses, 40 wins, and 59 LDWs. This reinforcement schedule (and payback percentage of $92 \%$ ) was based on the programming documents of a commercially available slot machine. All players received the same 301 spins hard coded in the same order.

As described in Dixon et al. (2019), six thought probes interrupted slots play after every 50 spins (although peripheral to the current new analyses, these are described below since theoretically they could have interrupted the experience of dark flow). Participants were prompted with a message asking about whether they were on-task (thinking about the game) or off-task (thinking about anything unrelated to the game) and to indicate their mood by pointing to a cartoon character that best approximated their current mood (self-assessment manikins; Bradley \& Lang, 1994). Responding to the thought probes (and pointing to the manikins) took approximately $5 \mathrm{~s}$, after which slots play was immediately resumed. Thought probes occurred after the 50th, 100th, 150th, 200th, 250th, and 300th spins. Following the $301 \mathrm{st}$ spin, a message indicated that players had $\$ 5.19$ remaining at the end of play, and that this amount would be rounded up to $\$ 10.00$.

Force transducer. A force transducer was fixed underneath the spin button of the slot-machine cabinet that housed the simulator. When depressed to initiate a spin, the amount of pressure applied to the spin button was translated to a volt signal recorded by $\mathrm{AD}$ Instruments PowerLabs (Colorado Springs, CO, USA) and saved in Labchart. Three separate slots simulators were used (each housed in a slot-machine casing with a force transducer mounted underneath the spin buttons).

\section{Materials}

Demographic questions. Participants completed demographic items on age and gender. Participants also completed an item derived from the Canadian Problem Gambling Index (Ferris \& Wynne, 2001) that assesses the frequency with which individuals engage in slot-machine gambling: "In the past 12 months, how often did you bet or spend money on slot machines or video lottery terminal's in a casino?"

Problem Gambling Severity Index (PGSI). In addition to the gambling frequency item, participants also completed the remaining nine items from the PGSI - a reliable screening tool for gambling problems in the general population (Cronbach's $\alpha$ of .84; Ferris \& Wynne, 2001). Participants responded to each item on a scale with either $0=$ never, $1=$ sometimes, $2=$ most of the time, or $3=$ almost always.

Game Experiences Questionnaire (GEQ). Participants also completed two subscales from the core version of the Game Experience Questionnaire (GEQ; IJsselsteijn et al., 2007) to assess their experience of the slot-machine session. Participants completed the positive affect and flow subscales of the GEQ, each consisting of five items. The flow items were: "I was fully occupied with the game," "I forgot everything around me," "I lost track of time," I was deeply concentrated in the game," and "I lost connection with the outside world." The positive affect items were: "I thought it was fun," "I felt happy," "I felt good," "I enjoyed it," and "I felt aroused/excited" (following the study of Dixon et al., 2019, the latter item was used instead of "I felt content"). Participants rated each item on a 5-point scale consisting of the following options: $0=$ not at all, $1=$ slightly, $2=$ moderately, $3=$ fairly, or $4=$ extremely. Items from each scale were summed to compute a score for positive affect and a score for dark flow. The full GEQ has demonstrated good reliability, with Cronbach's $\alpha$ values ranging from .71 to .89 for the various subscales included (Poels, de Kort, \& IJsselsteijn, 2007).

Depression, Anxiety, and Stress Scale (DASS). Following the slot-machine game session, participants completed the short version of the Depression, Anxiety, and Stress Scale (DASS-21; Lovibond \& Lovibond, 1995). The DASS-21 consists of three subscales with seven items per scale. Participants indicated their experience of depression ("I couldn't seem to experience any positive feeling at all"), anxiety ("I felt I was close to panic"), and stress ("I found it hard to wind down") over the past week with either $0=n o t$ at all, $1=$ some of the time, $2=$ a good part of the time, or $3=$ most of the time. A depression score was computed by adding the degree to which they endorsed each of the seven depression items (maximum score $=21$ ). The full DASS-21 has demonstrated good reliability (as measured by Cronbach's $\alpha .93)$, as has the depression subscale $(0.88$; Henry \& Crawford, 2005).

\section{Measures}

Post-reinforcement pauses. PRPs comprised the delay between the delivery of an outcome (i.e., the last reel stopping) and the player's initiation of the next spin, measured in milliseconds (ms). The simulator was configured to send event markers to an ADIntruments PowerLab for each outcome delivery and spin initiation, allowing us to calculate the temporal difference between these events. For each participant, PRPs were calculated for each outcome except for the first and last outcomes and all outcomes that immediately proceeded thought probes (all of these excluded outcomes were programmed to be regular losses). 
In total, PRPs were calculated for 194 losses, 59 LDWs, and 40 wins. PRPs as a function of win size were estimated by calculating the slope of PRP length for the various win sizes (which ranged from 0 to 1016 credits). Steeper slopes indicated greater reward reactivity.

Force responses. Prior to calculating the force responses, we excluded the first and last trials as well as all force responses for the spins preceding the thought probes (the seven losses described above). We then used an algorithm to determine the local maximum force value following each spin and defined a 1,100 ms window from this point. Within this window, the local minimum value was subtracted from the local maximum value to determine the force response. For each participant, the force responses following each of the 293 outcomes were calculated. Force as a function of win size was estimated by calculating the slope of force responses plotted over the various win sizes. Steeper slopes indicated greater reward reactivity.

\section{Procedure}

Each participant approached the experiment station situated in the front lobby of the casino, where they completed the aforementioned prescreen items. If eligible, they completed an informed consent form and were then escorted to a laptop computer (Lenovo model 4446-25U, Lenovo [Singapore] Pvt. Ltd., China) equipped with Qualtrics online survey software (Provo, UT, USA) to complete the first battery of questionnaires (i.e., demographic questions, PGSI, the 21-item DASS and, for purposes peripheral to this experiment, the Mindful Attention Awareness Scale). Following the completion of the questionnaires, they then began the slot-machine session of the experiment. A researcher explained the game features (e.g., number of lines played, bet size, number of spins, etc.) and the instructions for the in-game mind-wandering and affect probes. In total, the participant played 301 spins on the slot machine and were interrupted six separate times (every 50 spins). When a prompt was delivered, a researcher asked the participant whether they were thinking about the game or something else immediately before the prompt occurred; the researcher then recorded the participant's response on Qualtrics. Following this item, the researcher then asked the participant to indicate their emotional valence using the Self-Assesment Manikins (Bradley \& Lang, 1994) displayed on the slot-machine cabinet. The participant pointed toward the manikin that best described how they were feeling immediately before the prompt occurred, the manikins ranged from very positive to very negative. The participant then resumed the slot-machine game. This pattern was repeated for each of the six prompts until the end of the slot-machine session. Following the slot-machine session, participants then completed the remaining surveys on the Lenovo laptop (GEQ and DASS-21), as well as two other scales for purposes peripheral to this experiment (the flow while mind-wandering scale, and an item which asked participants to estimate how often they won more than they wagered on the slot-machine simulator). Once the experiment was completed, participants were given their remuneration from the slot-machine session (end balance of $\$ 5.19$ rounded up to $\$ 10.00$ for each participant) and a $\$ 25.00$ Walmart gift card, along with a feedback letter and responsible gambling resources.

\section{Ethics}

All methods and procedures were approved by the University of Waterloo's Office of Research Ethics (protocol number: 30716).

\section{RESULTS}

As reported in Dixon et al. (2019), in terms of problem gambling status, we used the interpretive categories of the PGSI suggested by Currie, Hodgins, and Casey (2013). Our sample consisted of 33 non-problem gamblers (PGSI score of 0), 47 low-risk gamblers (PGSI score ranging from 1 to 4), 23 moderate risk gamblers (PGSI score ranging from 5 to 7), and 26 problem gamblers (PGSI scores of 8 and above). Depression totals from the DASS-21 were characterized using the interpretive categories of the DASS-21 suggested by Lovibond and Lovibond (1995). The majority of participants $(n=97)$ fell within the normal range of depression (scores of 0 to 4), 16 participants were characterized with mild depression (totals of 5 or 6), 15 with moderate depression (totals between 7 to 10$), 1$ with severe depression (a total of 11), and 1 with extremely severe depression (a total depression score of 14).

Despite attempting to recruit players who played once per week or more, when filling out the slots frequency question, 19 players indicated that they played less often than once per week: 14 indicated that they played 2-3 times a month and 5 indicated that they played even less frequently. Of those who did play at least once per week, 41 indicated playing once per week, 61 indicated playing 2-6 times per week, and 7 reported playing daily.

For the PRP and Force measures, five participants had to be excluded because of technical problems with the Labchart recordings. Thus, analyses were based on the remaining 124 participants. The PRPs as a function of win size had slopes that ranged from -2 to 36.93 with a mean of 9.43 and a standard deviation of 5.80 . The force as a function of win size had slopes that ranged from -0.0014 to 0.00066 and had a mean of 0.000052 and a standard deviation of 0.00012 .

The two measures of reward reactivity were correlated with each other, $r(122)=.225, p=.012$. As predicted, the PRPs as a function of win size were significantly correlated with positive affect ratings, $r(122)=.187, p=.038$. Consistent with the notion that this is a non-problematic route to slots enjoyment, the PRP measure of reward reactivity was negatively correlated with PGSI scores, $r(122)=-.181$, $p=.044$, and was uncorrelated with depression scores, $r(122)=-.004, p=.97$. The force measure was also significantly correlated with positive affect ratings, $r(122)=.24$, $p=.006$, but like the PRP measure was not positively correlated with either PGSI scores, $r(122)=-.14, p=.13$, or depression scores, $r(122)=-.032, p=.73$.

Consistent with the notion that escape gambling is a problematic means to slots enjoyment, as reported in Dixon et al. (2019), dark flow was found to be significantly correlated with positive affect ratings, $r(127)=.63$, $p<.001$, but was also significantly correlated with PGSI ratings, $r(127)=.25, p=.004$, and depressive symptomatology, $r(127)=.289, p<.001$. A summary of these correlations appears in Table 1. 
Dixon et al.

Table 1. Zero-order correlations for key variables

\begin{tabular}{|c|c|c|c|c|c|c|}
\hline & PGSI & PRP slope & Force slope & Dark flow & Positive affect & Depression score \\
\hline 1. PGSI & - & & & & & \\
\hline 2. PRP slope & $-.181 *$ & - & & & & \\
\hline 3. Force slope & -.138 & $.225^{*}$ & - & & & \\
\hline 4. Dark flow & $.250 * *$ & .140 & .096 & - & & \\
\hline 5. Positive affect & -.039 & $.187^{*}$ & $.244^{* *}$ & $.627 * *$ & - & \\
\hline 6. Depression score & $.462 * *$ & -.004 & -.032 & $.289 * *$ & -.115 & - \\
\hline
\end{tabular}

Note. PGSI: Problem Gambling Severity Index; PRP: post-reinforcement pause; PRP slope: slope of post-reinforcement pauses over win size; Force slope: slope of force over win size; Dark flow: endorsement of flow items on the GEQ; Positive affect: endorsement of positive affect items on the GEQ; Depression score: endorsement of the depression items on the DASS- 21.

${ }^{*} p<.05 .{ }^{* *} p<.01$.

To assess whether dark flow ratings significantly accounted for unique positive affect variance (not accounted for by reward reactivity), we used hierarchical linear multiple regression to predict positive affect in which the measure of reward reactivity was entered first, followed by our measure of dark flow (PRPs and force measures were analyzed in separate models below).

\section{Hierarchical regression using PRPs as a measure of reward reactivity}

At Step 1, our PRP measure of reward reactivity led to an $R^{2}$ of .035 (3.5\% of positive affect variance was accounted for by PRP-based reward reactivity), $F(1,122)=4.421, p=.038$. At Step 2, adding the measure of dark flow increased $R^{2}$ to .410 - a significant change in $R^{2}$ of $.375, F(1,121)=76.97$, $p<.001$. In the full model, our PRP measure of reward reactivity [standardized $\beta=0.10$, squared semi partial correlation $=.010, \quad t(121)=1.42, \quad p=.16]$ failed to uniquely account for positive affect variance when dark flow was in the model. Dark flow did, however, account for a large portion of unique positive affect variance, [standardized $\beta=0.62$, squared semi-partial correlation $=.376$, $t(121)=8.77, p<.001]$.

\section{Hierarchical regression using force as a measure of reward reactivity}

At Step 1, our force measure of reward reactivity led to an $R^{2}$ of $.060(6 \%$ of positive affect variance was accounted for by force based reward reactivity), $F(1,122)=7.75$, $p=.006$. At Step 2, adding our measure of dark flow increased $R^{2}$ to $.434-$ a significant change in $R^{2}$ of $.375, F(1,121)=80.177, p<.001$. In the full model, both our force measure (standardized $\beta=0.186$, squared semi-partial correlation $=.034, t(121)=2.70, p=.008]$ and our measure of dark flow [standardized $\beta=0.62$, squared semi-partial correlation $=.375, t(121)=8.954$, $p<.001]$ each accounted for unique positive affect variance. A summary of these hierarchical multiple regressions appears in Table 2.

\section{DISCUSSION}

Our two measures of in-game reward reactivity were significantly correlated, and both were correlated with the positive affect experienced during the slot-machine session. Specifically, the more gamblers reacted to rewards, the greater their retrospective ratings of positive affect during the slots session.

Despite predicting the degree to which players enjoyed playing slots, these measures of reward reactivity were either unrelated to problem gambling severity, or in the case of the PRP measure, were negatively correlated with problem gambling severity. Although the latter negative correlation is intriguing, we are hesitant to make strong claims about this negative relationship. Unlike the relationship between our measures of reward reactivity and slots enjoyment, which showed converging evidence from both the PRP measure and the force measure, for negative correlations with problem gambling, we found this relationship only with the PRP measure and not with the force measure. As such, we do not have converging evidence for less problematic players showing greater reward reactivity. As such, a more conservative claim is that reward reactivity is unrelated to problem gambling status.

Although PRPs are a reasonable measure of reward processing, they are susceptible to several limitations. Wins

Table 2. Summary of multiple regressions

\begin{tabular}{|c|c|c|c|c|c|}
\hline Step & Independent variable & $R^{2}$ & Change in $R^{2}$ & $F$ of $R$ change & $p$ \\
\hline \multicolumn{6}{|c|}{ Predicting positive affect using PRP measure of reward reactivity and dark flow } \\
\hline 1. & Reward reactivity (PRP) & .035 & .035 & 4.421 & .038 \\
\hline 2. & Dark flow & .410 & .375 & 76.967 & $<.001$ \\
\hline \multicolumn{6}{|c|}{ Predicting positive affect using force measure of reward reactivity and dark flow } \\
\hline 1. & Reward reactivity (force) & .060 & .060 & 7.746 & .006 \\
\hline 2. & Dark flow & .410 & .375 & 80.177 & $<.001$ \\
\hline
\end{tabular}

Note. PRP: post-reinforcement pause. 
and losses on commercially available slot machines are associated with very different experiences. When players spin and lose, there is an absence of auditory and visual feedback. The machine simply waits for the player to spin again. When players spin, then gain credits, the machine generates both celebratory sounds and animations. Crucially, the larger the win amount, the more extensive the feedback. In the auditory domain, the larger the win, the longer the celebratory jingle (Dixon et al., 2012). As such, in commercially available slot machines, and in our simulator, which was designed to emulate them, longer PRPs following bigger wins might simply be attributable to slots players waiting until the winning song has finished before initiating the next spin (Dixon et al., 2012). Three different factors suggest that despite this possibility, PRPs are still a valuable way of gauging reinforcement. First, the relation between PRP length and win size can still be seen even if the PRP measurement is defined as the temporal duration between the end of the celebratory reinforcement and the initiation of the next spin (Templeton, Dixon, Harrigan, \& Fugelsang, 2015). That is, players tend to pause even after all auditory feedback has been completed with longer pauses following bigger wins. Second, even when the celebratory reinforcement stimuli that are artificially controlled by ensuring all post-win sounds and animations are the same length, the systematic relation between PRP size and win size can still be observed (Dixon et al., 2012). Furthermore, on commercially available machines (and simulators used in the experiment), players need not wait until the winning jingle has been completed - they can initiate the next spin during the celebratory feedback if they so choose. Finally, the PRP measure was significantly correlated with the force measure, and both the PRP and force measures accounted for significant positive affect variance. This suggests that despite its inherent problems as a measure, the PRPs indeed indexed the degree to which players enjoyed slot-machine wins. Despite these counterarguments, the fact that the length of the celebratory sounds varies systematically with win size makes the PRP less than ideal, leading us to place more interpretational weight on the force measure.

The force measure of reward reactivity was more strongly correlated with positive affect and accounted for greater unique positive affect variance in a multiple regression framework when dark flow was also in the model. Importantly, these measures (PRPs and force) of reward reactivity were unrelated to any of the negative associations of slots play (e.g., problem gambling or depression). As such, they appear to measure aspects of game enjoyment that are not related to problem gambling.

Our findings concerning dark flow stand in stark contrast to these measures of reward reactivity. First, dark flow accounted for far larger amounts of positive affect variance than the variance explained by reward reactivity. Second, unlike reward reactivity, dark flow experiences were strongly related to both problem gambling status and depression.

One contribution of this study lies in showing how reward reactivity and dark flow account for unique enjoyment variance. In Dixon et al. (2019), the authors showed how dark flow is associated with positive affect. We have rereported these correlations here in order to show how much more slots enjoyment variance is accounted for by the dark flow measure than by the reward reactivity measures. For example, the $6 \%$ of positive affect variance accounted for by our force measure of reactivity versus the $37.5 \%$ of addition unique variance accounted for by dark flow. In considering these magnitude differences, one must remember that in this sample there is a far larger proportion of moderate and problem gamblers than there would be in the general population. Since endorsements of dark flow are known to correlate with problem gambling, the fact that dark flow accounts for so much more positive affect variance may be in part because of this overrepresentation of problem players.

In Dixon et al. (2019), there were strong correlations between dark flow and both problem gambling and depression. We re-report these correlations here to contrast this problematic reason for enjoying slots, to the less problematic reasons for enjoying slots play related to the rewarding properties of the wins. Both our measures of reward reactivity were either uncorrelated or (slightly) negatively correlated with problem gambling and depression.

Another contribution is a methodological one. In this study, we report two new measures of reward reactivity that both gauge how much players enjoy playing slots. Unlike other psychophysiological measures involving cumbersome electrodes and wires (that likely reduce ecological validity and may inhibit flow), these measures are completely unobtrusive. They are gleaned from players who simply play the machines the way they would play their favorite multiline machines on the casino floor.

In summary, the degree to which participants reacted to rewards affected their enjoyment of slots play, but did not appear to be a risk factor for problem gambling. This reason for enjoyment stands in contrast to reasons for enjoying slots that are related to dark flow. We report strong positive correlations between dark flow and both depression and problem gambling status. These findings may have important implications for both the subtyping of problem gamblers and for the treatment of these gamblers. In the influential pathways model of problem and pathological gambling (Blaszczynski \& Nower, 2002), one pathway involves behavioral conditioning. In this group, arousal responses to gambling outcomes play a key role. Presumably early in one's gambling career, reward-related arousal responses are substantial, but, according to the model, diminish as tolerance develops. Players who habituate to reward-related arousal may gravitate to machines that allow greater wagers-per-spin to experience the same degree of reward reactivity. In this study, our measures of reward reactivity presumably could differ depending on the tolerance of players. Here, we found either no relation with problem gambling status as measured by the PGSI or a slight negative correlation. Recall that we recruited highfrequency players (at least once per week) in order to ensure the sampling of moderate and problem players. Had we tested both frequent players (potentially habituated to reward reactivity) and more infrequent players (who might show even higher reward reactivity), we might have expected even stronger negative correlations between reward reactivity and problem gambling severity.

Whether such negative correlations would be found might depend on the specificity of the celebratory feedback 
to which players become conditioned. It is an empirical question whether players might become conditioned to (and habituate to) the exact jingles of their favorite machines or whether players would show conditioned responses to a "class" of celebratory feedback like the jingles and animations used in the simulators. One future direction would be to compare the PRPs and force measures of reward reactivity of players at different stages of their gambling careers playing on familiar machines. One might expect that as players become habituated, the magnitude of these reward reactivity metrics would decline.

The second route to gambling problems proposed in the pathways model involves emotionally vulnerable individuals who may gamble to "escape." In this study, we show strong correlations between both depression scores and problem scores with our measures of dark flow. Importantly, we show that the degree to which participants endorsed dark flow was very strongly related to the positive affect they reported during the slots session. In Dixon et al. (2019), we proposed that depressed players may have a hard time keeping their thoughts on whatever day-to-day tasks they are doing, but rather have a propensity to mind-wander, with their thoughts straying toward negative mentations. Multiline slot machines with their high celebratory event frequency may serve to rein in these wandering minds and induce dark flow. Crucially, such flow states are strongly related to positive affect. Thus, the high degree of positive affect reported by those experiencing dark flow may reflect the negatively reinforcing properties of slot-machine play for this subgroup. They play not for the so called "arousal high" associated with reward reactivity, but for the relief that comes from not thinking about the negative aspects of their lives during the dark flow state. In terms of treatment implications, teaching problem gamblers to be more mindful in everyday life (to stay on-task, and curtail mind-wandering) may be a particularly effective treatment option for those who gamble on slot machines to escape (Chen, Jindani, Perry, \& Turner, 2014).

The pathways model provides an exposition of the different routes players take in their development of problem gambling. As noted above, our reward reactivity measures and dark flow may be relevant to these different pathways with reward reactivity related to the behavioral conditioning pathway and dark flow related to the pathway taken by emotionally vulnerable people. The pathways model presumes that a given gambler follows one pathway or another. Our measures are related to two different means to enjoying slots and it is possible that the same person could experience any combination of reward reactivity and dark flow. That is, the same person might have two different reasons for enjoying slots indeed, the fact that dark flow accounts for positive affect variance that is distinct from reward reactivity underscores that there are multiple ways in which players can enjoy slots.

In conclusion, we present evidence that suggests that there are two different means to enjoying slot-machine play. One route to enjoyment appears far more problematic than the other. While reacting more strongly to wins is not associated with gambling problems, experiencing dark flow is related to problem gambling status and depression. Thus enjoying slots play via dark flow may be reflective of using slot-machine play to escape from the harsh realities experienced by these depressed and problem players.
Funding sources: This research was funded by the Manitoba Gambling Research Program of Manitoba Liquor \& Lotteries; however, the findings and conclusions of this paper are those solely of the authors and do not necessarily represent the views of Manitoba Liquor \& Lotteries. The funding sponsor was not involved in the study design, collection, analysis or interpretation of the data, the writing of the manuscript, and the decision to submit the manuscript for publication.

Authors' contribution: Each author contributed to the initial ideas. MJD, JG, and TBK were involved in writing of the manuscript. CJL, MS, CG, and SDS were involved with the consultation of the design and framing the findings.

Conflict of interest: The authors declare no conflict of interest.

Acknowledgements: The authors would like to acknowledge Rose Gregorio and her Responsible Gambling Team at the Brantford Casino.

\section{REFERENCES}

Abbot, M. W., \& Volberg, R. A. (1996). The New Zealand national survey of problem and pathological gambling. Journal of Gambling Studies, 12, 143-160. doi:10.1007/BF01539171

Anderson, G., \& Brown, R. I. (1984). Real and laboratory gambling, sensation-seeking and arousal. British Journal of Psychology, 75(3), 401-410. doi:10.1111/j.2044-8295.1984.tb01910.x

Blaszczynski, A., \& Nower, L. (2002). A pathways model of problem and pathological gambling. Addiction, 97(5), 487-499. doi:10.1046/j.1360-0443.2002.00015.x

Blaszczynski, A., Sharpe, L., Walker, M., Shannon, K., \& Coughlan, M. J. (2005). Structural characteristics of electronic gaming machines and satisfaction of play among recreational and problem gamblers. International Gambling Studies, 5(2), 187-198. doi:10.1080/14459790500303378

Bradley, M. M., \& Lang, P. J. (1994). Measuring emotion: The self-assessment manikin and the semantic differential. Journal of Behavior Therapy and Experimental Psychiatry, 25(1), 49-59. doi:10.1016/0005-7916(94)90063-9

Brown, R. I. F. (1986). Arousal and sensation-seeking components in the general explanation of gambling and gambling addictions. International Journal of the Addictions, 21(9-10), 1001-1016. doi:10.3109/10826088609077251

Chen, P., Jindani, F., Perry, J., \& Tyrberm, N. L. (2014). Mindfulness and problem gambling treatment. Asian Journal of Gambling Issues and Public Health, 4(2). doi:10.1186/ 2195-3007-4-2

Csikszentmihalyi, M. (1992). Flow: The psychology of happiness. London, UK: Rider.

Coulombe, A., Ladouceur, R., Desharnais, R., \& Jobin, J. (1992). Erroneous perceptions and arousal among regular and occasional video poker players. Journal of Gambling Studies, 8(3), 235-244. doi:10.1007/BF01014651

Coventry, K. R., \& Constable, B. (1999). Physiological arousal and sensation-seeking in female fruit machine gamblers. Addiction, 94(3), 425-430. doi:10.1046/j.1360-0443.1999.94342512.x 
Coventry, K. R., \& Hudson, J. (2001). Gender differences, physiological arousal and the role of winning in fruit machine gamblers. Addiction, 96(6), 871-879. doi:10.1046/j.13600443.2001.9668718.x

Coventry, K. R., \& Norman, A. C. (1998). Arousal, erroneous verbalizations and the illusion of control during a computer-generated gambling task. British Journal of Psychology, 89(4), 629-645. doi:10.1111/j.2044-8295.1998.tb02707.x

Currie, S. R., Hodgins, D. C., \& Casey, D. M. (2013). Validity of the Problem Gambling Severity Index interpretive categories. Journal of Gambling Studies, 29(2), 311-327. doi:10.1007/ s10899-012-9300-6

Delfabbro, P. H., \& Winefield, A. H. (1999). Poker-machine gambling: An analysis of within session characteristics. British Journal of Psychology, 90(3), 425-439. doi:10.1348/000712 699161503

Diskin, K., \& Hodgins, D. C. (1999). Narrowing of attention and dissociation in pathological video lottery gamblers. Journal of Gambling Studies, 9, 225-245.

Diskin, K. M., \& Hodgins, D. C. (2001). Narrowed focus and dissociative experiences in a community sample of experienced video lottery gamblers. Canadian Journal of Behavioural Science, 33(1), 58-64. doi:10.1037/h0087128

Diskin, K. M., \& Hodgins, D. C. (2003). Psychophysiological and subjective arousal during gambling in pathological and nonpathological video lottery gamblers. International Gambling Studies, 3(1), 37-51. doi:10.1080/14459790304590

Dixon, M. J., Graydon, C., Harrigan, K. A., Wojtowicz, L., Siu, V., \& Fugelsang, J. A. (2014). The allure of multi-line games in modern slot machines. Addiction, 109(11), 1920-1928. doi:10.1111/add.12675

Dixon, M. J., Gutierrez, J., Stange, M., Larche, C. J., Graydon, C., Vintan, S., \& Kruger, T. B. (2019). Mindfulness problems and depression symptoms in everyday life predict dark flow during slots play: Implications for gambling as a form of escape. Psychology of Addictive Behaviors, 33(1), 81-90. doi:10.1037/ adb0000435

Dixon, M. J., Harrigan, K. A., Jarick, M., MacLaren, V. V., Fugelsang, J. A., \& Sheepy, E. (2011). Psychophysical arousal signatures of near-misses in slot machine play. International Gambling Studies, 11(3), 393-407. doi:10.1080/144597 95.2011.603134

Dixon, M. J., Harrigan, K. A., Sandhu, R., Collins, K., \& Fugelsang, J. A. (2010). Losses disguised as wins in modern multiline video slot machines. Addiction, 105(10), 1819-1824. doi:10.1111/j.1360-0443.2010.03050.x

Dixon, M. J., Harrigan, K. A., Santesso, D. L., Graydon, C., Fugelsang, J. A., \& Collins, K. (2014). The impact of sound in modern multiline video slot machine play. Journal of Gambling Studies, 30(4), 913-929. doi:10.1007/s10899-013-9391-8

Dixon, M. J., Larche, C. J., Stange, M., Graydon, C., \& Fugelsang, J. A. (2017). Near-misses and stop buttons in slot machine play: An investigation of how they affect players, and may foster erroneous cognitions. Journal of Gambling Studies, 34(1), 161-180. doi:10.1007/s10899-017-9699-x

Dixon, M. J., Maclaren, V., Jarick, M., Fugelsang, J. A., \& Harrigan, K. A. (2012). The frustrating effects of just missing the jackpot: Slot machine near-misses trigger large skin conductance responses, but no post-reinforcement pauses. Journal of Gambling Studies, 29(4), 661-674. doi:10.1007/ s10899-012-9333-x
Dixon, M. J., Stange, M., Larche, C., Graydon, C., Fugelsang, J., \& Harrigan, K. (2017). Dark flow, depression and multiline slot machine play. Journal of Gambling Studies, 34(1), 73-84. doi:10.1007/s10899-017-9695-1

Dixon, M. R., \& Schreiber, J. E. (2004). Near-miss effects on response latencies and win estimations of slot machine players. The Psychological Record, 54(3), 335-348. doi:10.1007/ BF03395477

Ferris, J. A., \& Wynne, H. J. (2001). The Canadian Problem Gambling Index. Ottawa, ON: Canadian Centre on Substance Abuse.

Getty, H. A., Watson, J., \& Frisch, G. R. (2000). A comparison of depression and styles of coping in male and female GA members and controls. Journal of Gambling Studies, 16(4), 377-391. doi:10.1023/A:1009480106531

Griffiths, M., \& Parke, J. (2005). The psychology of music in gambling environments: An observational research note. Journal of Gambling Issues, 13. doi:10.4309/jgi.2005.13.8

Haas, E. C., \& Edworthy, J. (1996). Designing urgency into auditory warnings using pitch, speed and loudness. Computing and Control Engineering Journal, 7(4), 193-198. doi:10.1049/ cce: 19960407

Haw, J. (2008). The relationship between reinforcement and gaming machine choice. Journalof Gambling Studies, 24(1), 55-61. doi:10.1007/s10899-007-9073-5

Henry, J. D., \& Crawford, J. R. (2005). The short-form version of the Depression Anxiety Stress Scales (DASS-21): Construct validity and normative data in a large non-clinical sample. British Journal of Clinical Psychology, 44(2), 227-239. doi:10.1348/014466505X29657

IJsselsteijn, W., de Kort, Y., Poels, K., Jurgelionis, A., \& Bellotti, F. (2007, June 13-15). Characterising and measuring user experiences in digital games. Paper presented atProceedings of the International Conference on Advances in Computer Entertainment Technology (ACE 2007), Salzburg, Austria. Retrieved from http://www.webcitation.org/6Rfx7u6SI. Accessed on: August 8, 2014.

Jacobs, D. F. (1988). Evidence for a common dissociative-like reaction among addicts. Journal of Gambling Behaviour, 4(1), 27-37. doi:10.1007/BF01043526

Lahn, J. (2005). Gambling among offenders: Results from an Australian survey. International Journal of Offender Therapy and Comparative Criminology, 49(3), 343-355. doi:10.1177/ $0306624 X 04270790$

Leslie, J. C. (1996). Principles of behaviour analysis. Amsterdam: Harwood Academic Publishers.

Lole, L., Gonsalvez, C. J., Blaszczynski, A., \& Clarke, A. R. (2011). Electrodermal activity reliably captures physiological differences between wins and losses during gambling on electronic machines. Psychophysiology, 49(2), 154-163. doi:10.1111/j.1469-8986.2011.01290.x

Lovibond, P. F., \& Lovibond, S. H. (1995). The structure of negative emotional states: Comparison of the Depression Anxiety Stress Scales (DASS) with the Beck Depression and Anxiety Inventories. Behaviour Research and Therapy, 33(3), 335-343.

Meyer, G., Schwertfeger, J., Exton, M. S., Janssen, O. E., Knapp, W., Stadler, M. A., Schedlowski, M., \& Krüger, T. H. (2004). Neuroendocrine response to casino gambling in problem gamblers. Psychoneuroendocrinology, 29(10), 1272-12800. doi:10.1016/j.psyneuen.2004.03.005 
Murch, W. S., Chu, S., \& Clark, L. (2017). Measuring the slot machine zone with attentional dual tasks and respiratory sinus arrhythmia. Psychology of Addictive Behaviors, 31(3), 375-384. doi:10.1037/adb0000251

Paris, J. J., Franco, C., Sodano, R., Frye, C. A., \& Wulfert, E. (2010). Gambling pathology is associated with dampened cortisol response among men and women. Physiology \& Behavior, 99(2), 230-233. doi:10.1016/j.physbeh.2009. 04.002

Poels, K., de Kort, Y. A. W., \& IJsselsteijn, W. A. (2007). D3.3: Game Experience Questionnaire: Development of a self-report measure to assess the psychological impact of digital games. Eindhoven, The Netherlands: Technische Universiteit Eindhoven.

Schüll, N. D. (2005). Digital gambling: The coincidence of desire and design. The Annals of the American Academy of Political and Social Science, 597(1), 65-81. doi:10.1177/000271 6204270435
Schüll, N. D. (2012). Addiction by design: Machine gambling in Las Vegas. Princeton, NJ: Princeton University Press.

Schluter, M. G., \& Hodgins, D. C. (2019). Dissociative experiences in gambling disorder. Current Addiction Reports, 6(1), 34-40. doi:10.1007/s40429-019-0238-y

Sharpe, L., Tarrier, N., Schotte, D., \& Spence, S. H. (1995). The role of autonomic arousal in problem gambling. Addiction, 90(11), 1529-1540. doi:10.1111/j.1360-0443.1995.tb02815.x

Sodano, R., \& Wulfert, E. (2010). Cue reactivity in active pathological, abstinent pathological, and regular gamblers. Journal of Gambling Studies, 26(1), 53-65. doi:10.1007/ s10899-009-9146-8

Templeton, J. A., Dixon, M. J., Harrigan, K. A., \& Fugelsang, J. A. (2015). Upping the reinforcement rate by playing the maximum lines in multi-line slot machine play. Journal of Gambling Studies, 31(3), 949-964. doi:10.1007/s10899-014-9446-5

Walker, M. (1992). The psychology of gambling. Oxford, UK: Pergamon Press. 